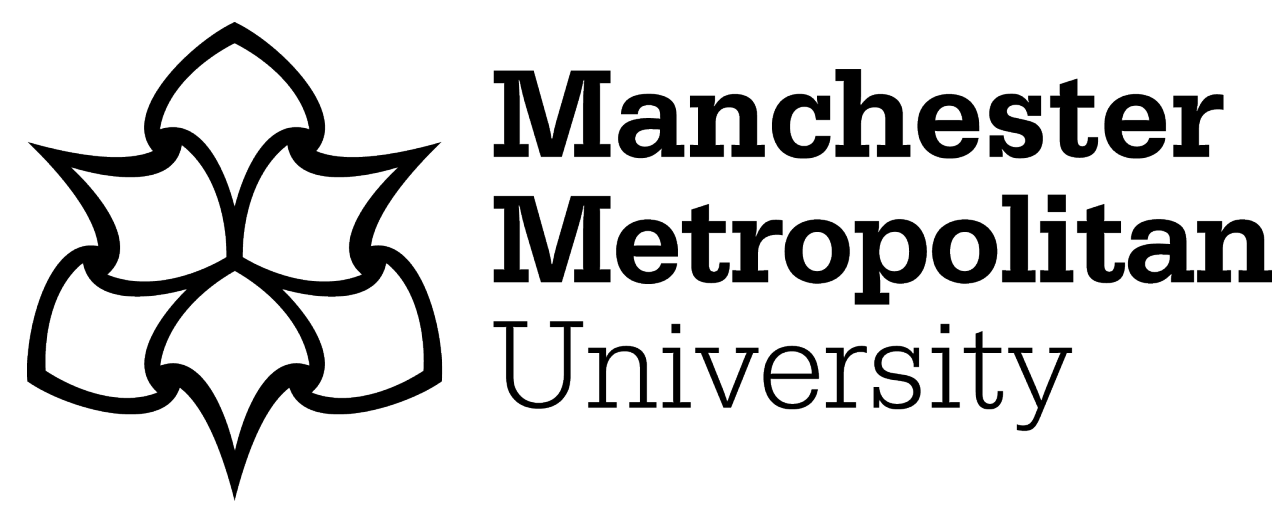

Stamou, Vasileios, Chatzoudi, Theano, Stamou, Lelouda, Romo, Lucia and Graziani, Pierluigi (2016) Music-assisted systematic desensitization for the reduction of craving in response to drug-conditioned cues: A pilot study. The Arts in Psychotherapy, 51. pp. 36-45. ISSN 0197-4556

Downloaded from: https://e-space.mmu.ac.uk/625446/

Version: Accepted Version

Publisher: Elsevier BV

DOI: https://doi.org/10.1016/j.aip.2016.08.003

Please cite the published version 


\title{
Music-assisted systematic desensitization for the reduction of craving in response to drug-conditioned cues: a pilot study.
}

\begin{abstract}
In the last decades, increasing research findings regarding the therapeutic value of music have led music-assisted therapeutic interventions to be more frequently considered by clinicians as complementary modalities for drug addiction treatment. We hypothesized that two different music modalities combined with systematic desensitization can counter-condition drugrelated cues and significantly reduce the implicated craving responses. We further examined the effect of the two treatments on cognitive and psychopathological components of addiction. Twenty-four drug addicted individuals were randomly assigned to one of the three study groups, namely systematic desensitization (SD) combined with listening to New Age meditation music improvised live on Tibetan bowls (IMT), systematic desensitization combined with listening to relaxing New Age music recorded in individual MP3 devices (NIMT), and control group that received no additional therapeutic intervention (CTR). Participants of the two treatment groups received a six-session therapy in addition to their standard treatment during a period of three weeks. Evaluation took place at baseline, posttreatment and one month after the end of treatment. Results showed that IMT and NIMT combined with SD significantly reduced craving in response to external drug-conditioned cues, while IMT appeared to be slightly more effective than NIMT in altering permissive thoughts on drug and alcohol use, depression symptoms and everyday life craving reactivity. Further investigation on the role of music-assisted systematic desensitization as a therapeutic intervention for drug addiction treatment seems warranted.
\end{abstract}

Key words: music, craving, drugs, addiction, cue-exposure therapy, systematic desensitization 


\section{Introduction}

During the past decades the disease model has been one of the most dominant theoretical frameworks of addiction. This model argues that individual physiology and genetics are responsible for the development of addiction (Hall, Carter, \& Forlini, 2015). However, as research studies have not managed to substantiate the validity of this theory (Levy, 2013), the scientific focus has moved on other models, such as the cognitive behavioural theory of addiction which posits that drug addiction is a learned behaviour that mainly takes place via association. This notion is partly based on the Classical Conditioning Theory (Pavlov, 1927/1960) according to which previously neutral external or internal stimuli can become conditioned cues if repeatedly paired with drug use (Myers \& Carlezon, 2010; Ramirez, Monti, \& Colwill, 2015). Subsequent exposure to these cues induces conditioned psychophysiological, subjective and behavioural responses that increase the motivation for maintaining drug use and consequently the risk for relapse (Hone-Blanchet, Wensing, \& Fecteau, 2014).

One of the therapeutic objectives of Cognitive Behavioural Therapy (CBT) is to timely identify high-risk situations that involve drug-conditioned cues and provide individuals suffering from substance use disorders (SUD) with alternative coping strategies in order to sustain abstinence (Yokoyama et al., 2015). This is mainly achieved via cueexposure therapeutic interventions that aim at extinguishing drug-conditioned cues and the implicated craving responses (Hone-Blanchet, Wensing, \& Fecteau, 2014). Cue-exposure therapeutic interventions have been widely employed for drug addiction treatment (Manley, 2008) but have produced contradictory results (McHugh, Park, \& Weiss, 2014; Romo \& Graziani, 2015). The main therapeutic target in the majority of the research studies has been the extinction of drug-conditioned cues, and consequently the weakening of the association between cues and psychophysiological responses related to craving. However, contemporary 
views argue that according to Pavlovian theories, repeated unreinforced exposure to the cues can only conceal the association rather than weaken it (Van Gucht, Baeyens, Hermans, \& Beckers, 2013). Consequently, the notion of cue extinction as a form of unlearning maladaptive behaviours has been challenged and replaced by the notion of cue counterconditioning, which proposes learning a new behaviour in response to the cue and developing a new association that will promote healthy behavioural patterns and will facilitate abstinence (Van Gucht, Baeyens, Vansteenwegen, Hermans, \& Beckers, 2010).

The counterconditioning of drug-conditioned cues necessitates the existence of an alternative emotion-inducing source that will facilitate counteracting the psychophysiological responses related to craving and will allow the modification of the implicated drug-seeking behaviours. Although there are numerous views on drug craving, it is predominantly considered as an intense desire with strong affective properties (May, Kavanagh, \& Andrade, 2015). It is often mediated or preceded by specific psychophysiological responses to internal or external stimuli (Tiffany, 2010; Andrade, May, \& Kavanagh, 2008), in anticipation to the positive effect of the substance or to relief from withdrawal symptoms. These processes occur very rapidly and often with little or no awareness at all by the individual involved (Andrade, May, \& Kavanagh, 2008). Consequently, it may be argued that counteracting the psychophysiological effects induced by such stimuli may facilitate the decrease of craving, as well as the interruption and modification of the implicated drug-seeking behavioural patterns. This may be achieved by the induction of positive emotions, a mechanism that has been proposed for counteracting the psychophysiological effects evoked by negative affective states (Sheldon, Kashdan, \& Steger, 2011).

Importantly, music listening has been proposed as a source for eliciting distinct emotions of positive valence (Salimpoor, Benovoy, Larcher, Dagher, \& Zatorre, 2011; 
Salimpoor, Benovoy, Longo, Cooperstock, \& Zatorre, 2009) that can counteract negative affect (Phipps, Carroll, \& Tsiantoulas, 2010), and enhance psychophysiological homeostatic functions (Bernatzky, Presch, Anderson, \& Panksepp, 2011; Sakamoto, Ando, \& Tsutou, 2013). For instance, it has been shown that listening to relaxing music can counteract the negative effects of stress and increase relaxation after exposure to a stressor (Labbe, Schmidt, Babin, \& Pharr, 2007). Recent neurobiological research findings attribute the aforementioned effects of music to music-induced dopamine release in the brain's mesolimbic system (Menon \& Levitin, 2005; Salimpoor et al., 2011) and to deactivation of the brain hippocampus and amygdala that are involved in the experience of negative emotions (Blood \& Zatorre, 2001). This proposed mechanism may partly explain why individuals with SUD intentionally listen to music in an effort to alleviate physical and psychological pain and discomfort (Aldridge \& Fachner, 2006), such as that induced by depression (Cevasco, Kennedy, \& Generally, 2005; Mays, Clark, \& Gordon, 2008). In addition, music can act as a mild auditory cue for emotions in individuals with SUD (Short \& Dingle, 2015) and facilitate the experience of moderate or intense positive emotions without the need for substances, while allowing the exploration of negative emotions in a safe environment (Baker, Gleadhill, \& Dingle, 2007). The above findings imply that music may be useful as a means for inducing positive emotions in order to counteract the psychophysiological effects evoked by negative affective states, such as craving.

On the other hand, music may also carry a risk for relapse. It has been reported that music associated with drug use can elicit craving and consequently increase the possibility for relapse (Dingle, Kelly, Flynn, \& Baker, 2015). However, the effect of music combined with a treatment modality that attenuates drug-seeking behaviours has not been tested. It could, therefore, be argued that listening to relaxing music that has not been formerly 
associated with drug use may be capable of facilitating the counterconditioning of drugconditioned cues and enhancing the effectiveness of cue-exposure therapy in reducing the implicated craving responses. Such a contribution may prove to be significant for providing individuals with SUD with efficient coping strategies for high-risk situations that involve substance-related cues. These strategies may eventually promote problem-based coping via the reacquisition of cognitive control over cue reactivity and the containment of compulsive substance-seeking behaviour in the presence of drug-related stimuli (Lee et al., 2013).

Permissive thoughts have been particularly highlighted for their involvement in the responsivity to substance-conditioned cues (Romo \& Graziani, 2015). CBT argues that treatment must involve the addicted individual's control over his/her permissive thoughts and the modification of them in a therapeutically beneficial manner. Permissive thoughts, such as "If I take drugs just once more it won't hurt me, I can handle it", concern the way the addicted individual perceives substance use as acceptable despite his/her awareness of the negative consequences. This is mainly due to the perception of substance use as an efficient means for coping with situations or internal states that place demands beyond an individual's capacity to cope (Havermans \& Jansen, 2003; Stathopoulou, 2009). Repeated drug use subsequently leads to the conditioning of the situations that act as cues and result in automatic behavioural responses of drug use. These cues induce high levels of craving and negative affect when the substance is not available (Wise \& Koob, 2014). During this process, permissive thoughts promote the use of substances in search for alleviating craving and the implicated psychophysiological responses (Romo \& Graziani, 2015). Thus, it seems reasonable to assume that counter-conditioning substance-related cues could result in the reduction of permissive thoughts in the presence of related stimuli. Of importance, music has shown to counteract negative ideas experienced in stressful environments (Phipps et al., 
2010) and of enhancing positive beliefs that outlast individual tendencies to counteract them (Salamon, Kim, Beaulieu, \& Stefano, 2003). It can be, therefore, assumed that this property of music may be useful in facilitating synergistically with cue-exposure therapy the decrease of permissive thoughts of individuals with SUD.

Depression is another factor strongly associated with drug use (Saban, Flisher, Laubscher, London, \& Morojele, 2014). The main notion behind this association is that drug use serves as a means for alleviating pre-existing depression or depressive symptoms that drug abuse actually causes (Ersche, Turton, Pradhan, Bullmore, \& Robbins, 2010), which results in a vicious cycle of one condition negatively affecting the other (Saban et al., 2014). Notably, both cue-exposure therapy and music have been found to facilitate the reduction of depression symptoms in individuals with SUD (Cevasco et al., 2005; Emmelkamp \& Vedel, 2012) and their combination could potentially produce an enhanced therapeutic effect.

The above findings indicate the potential role of music in enhancing coping skills of individuals with SUD via counter-conditioning cues associated with drug use and reducing related craving responses. In this pilot study we examined the effectiveness of in vitro cueexposure therapy combined with two different modalities of relaxing music (de Niet, Tiemens, Lendemeijer, \& Hutschemaekers, 2009; Smith \& Joyce, 2004) not formerly associated with drug use, in facilitating the rehabilitation of individuals with SUD. We hypothesized that systematic desensitization combined with listening to New Age meditation music improvised live on Tibetan bowls or listening to New Age relaxing music recorded in individual MP3 devices, can reduce craving intensity during exposure to external drugconditioned cues. These two music modalities were chosen due to their capacity to induce feelings of relaxation (Landry, 2014; Serafin, Wilkerson, \& Smith, 2002; Kemper \& Danhauer, 2005) that were expected to facilitate counter-conditioning substance-associated 
cues. We further aimed at investigating the effect of the proposed treatments on craving reactivity in everyday life, permissive thoughts on drug and alcohol use, and depression symptoms.

\section{Materials and Methods}

The study received approval from the Aix-Marseille University Ethics Committee and from the Commission of Informatics, Liberty and Data Protection in France. The study described in the context of this paper was conducted in the ARGO Alternative Therapeutic Program for Addicted Individuals in the Psychiatric Hospital of Thessaloniki, Greece and was supported by a Grundtvig assistantship that was awarded to the first author by the Grundtvig Education and Culture Lifelong Learning Program for implementing such research in Greece (agreement contract number 2013-1-FR1-GRU11-50690).

\subsection{Research setting and participants}

The study was conducted in the ARGO Alternative Therapeutic Program for Addicted Individuals which is a substance-free non-residential therapeutic program. ARGO belongs to the National Health System of Greece and specifically to the General Hospital of Thessaloniki "G. Papanikolaou” - Unit of Psychiatric Hospital of Thessaloniki, in Greece. It deals with addictions from any legal or illegal substance and offers services to people of 18 years of age and above. The duration of treatment in ARGO varies from 28 to 36 months in total and is composed of three different stages: a) the Counselling Station, that mainly consists of intake assessment, motivational interviews, detoxification consultation, dispensation of opium antagonists if needed, health check-ups, and therapy groups for personal support and motivation, abstinence maintenance and family support (total duration 2 months), b) the Therapeutic Community, which is the most intense treatment period and is composed of daily individual and group therapy sessions, such as 24-hours review and 24- 
hours preparation, weekend-preview and weekend-preparation, behavioural reconstruction group, experiential group, reflectional thought group, photogram group, corporal expression group and psychodrama (total duration 18 months), and c) the Social Reintegration Unit, which is composed of psychosocial support groups and sports and cultural activities that take place twice a week (total duration 8 to 16 months, depending on therapeutic progress).

Twenty four individuals who were diagnosed with drug dependence according to the ICD 10 (World Health Organization, 1990) and received regular treatment in the Therapeutic Community of ARGO program participated in the study. According to the research protocol, individuals suffering from co-occurring schizophrenia, psychosis or uncontrolled bipolar disorder could not be included in the study due to the administration of medication that could alter the levels of craving. Such condition would interfere with the protocol of the study and possibly affect the results. However, none of the 24 participants met the exclusion criteria and were, therefore, all included in the sample.

\subsection{Procedure}

\subsubsection{Pre-treatment phase}

The therapeutic modalities investigated in the context of this study substituted one of the optional therapy groups of ARGO program. Potential participants were initially debriefed on the purpose, objectives and design of the study. It was highlighted that participation was voluntary and participants could retire from the study at any time without any consequences for them or the treatment they received in the ARGO program. Subsequently, 24 drugdependent individuals provided their written consent in order to participate in the study.

The randomization of the sample was ensured via a draw that took place in front of all participants. Small pieces of paper that contained the name of each participant were randomly 
chosen in order to assign them to one of the following groups: a) eight participants for the Instrumental Music Therapy group (IMT) who would receive systematic desensitization (SD) combined with listening to slow-paced low-pitched meditation music without lyrics improvised live on Tibetan bowls, in addition to their standard treatment, b) eight participants for the Non-Instrumental Music Therapy group (NIMT) who would receive systematic desensitization combined with listening to slow-paced low-pitched New Age relaxing music without lyrics recorded in individual MP3 devices, in addition to their standard treatment, and c) eight participants for the control group (CTR) who would receive no therapeutic intervention in addition to their standard treatment. At the end of the randomization process, all participants were aware of the group to which they were assigned, as well as of the groups to which the rest of the participants were assigned. The CTR group was informed that six music-assisted systematic desensitization sessions would be offered to any of the members who wished so, after the end of the research study. This was done to ensure that the CTR group members' motivation for participation and consequently their scores during the measures would not be affected.

Participants assigned to IMT and NIMT groups attended an individual interview during which they were asked to identify three situations of everyday life that they considered as high-risk for relapse due to the presence of drug-conditioned cues. The three situations were chosen by the participants with the aid of the researchers based on the criteria of frequency and craving intensity. Following, hierarchies were collaboratively built for gradual exposure to each situation by the researchers and the participants. Subsequently, the therapeutic process of each session was fully explained to each participant and a step-by-step written guide was given to them, so that they could fully comprehend the therapeutic course 
for counter-conditioning each situation. Participants were also informed that they would receive further guidance and clarifications during the therapeutic sessions.

The baseline assessment consisted of participants filling out a socio-demographic questionnaire, three self-report questionnaires and a craving evaluation during exposure to external drug-conditioned cues. The craving evaluation process involved a fifteen-minute film projection that included the most salient drug-conditioned cues identified by participants during the individual interviews, namely drug paraphernalia, drug use, drug dealing and pharmacy signs.

Participants of the NIMT group were then presented with 3 pieces of New Age relaxing music entitled "Calming eastern temple of clarity", "Enchanted garden of peace" and "Soothing sea of tranquillity", composed by Hot Yoga Masters and of 40 minutes duration each. They were asked to choose the piece or pieces they preferred to use during the phase of treatment. These three musical pieces shared the same musical characteristics with the IMT music modality, namely slow tempo, low-pitch sounds and the absence of lyrics.

\subsubsection{Treatment phase}

IMT and NIMT participants received a 3-week treatment that consisted of 2 therapeutic sessions per week for each group. Each high-risk situation was counterconditioned in two therapeutic sessions.

IMT sessions began with a brief explanation of the session by the researchers during which participants could also ask for clarifications. Following, participants listened to the New Age meditation music improvised live on Tibetan bowls by a professional musician for 20 minutes, in order to relax. Subsequently, they were imaginarily exposed to the high-risk situation for 50 minutes, following the gradual steps of the hierarchy and the written step-by- 
step guide built during the individual interviews. During imaginary exposure, the professional musician continued to play the music to facilitate the counterconditioning of the high-risk situation and the modification of the psychophysiological responses that were associated with the accompanying craving. Every 10 minutes participants were signalled to evaluate the intensity of their craving in a scale from 0 to 100 . Each session ended with a 10 -minute feedback during which participants discussed their experience with the researchers.

NIMT sessions followed the exact same process but instead of listening to meditation music improvised live on Tibetan bowls, participants listened to the New Age music selection they had chosen in the pre-treatment phase in individual MP3 devices. During the therapeutic sessions of both IMT and NIMT groups, the researchers were available to provide guidance and clarifications when needed.

In case of craving urges persisting after the end of the sessions, the researchers and members of the ARGO therapeutic team stayed with participants until the urges completely faded out in order to eliminate any possibility for relapse.

\subsubsection{Post-treatment phase}

The baseline measurements were repeated immediately after the end of the treatment phase and at a follow-up visit that took place 1 month later. Participants were notified of their right to obtain their personalized data produced during these measurements. After the followup, each participant could obtain this data upon request.

\subsection{Outcome Assessments}

The baseline and post-treatment evaluations were conducted using the following instruments: 


\section{i. Symptoms Checklist-90-R Derogatis (SCL-90-R)}

Symptoms Checklist 90 (Derogatis, 1993) is a screening instrument that measures ten categories of psychopathological symptoms, including depression. Although these are rather symptom dimensions and are not corresponding to a direct diagnosis based on ICD-10, the Symptoms Checklist 90 is considered as one of the most reliable and widely employed instruments for measuring a treatment effect on the depression symptoms of individuals with SUD (Deady, 2009). The Symptoms Checklist 90 is normed on adult psychiatric outpatients, non-patients and psychiatric inpatients, as well as on adolescent non-patients. The instrument's internal consistency reliability (Cronbach's alpha) in measuring depression has been estimated at .9 (Horowitz, Rosenberg, Baer, Ureno, \& Villasenor, 1988).

\section{ii. Craving Reactivity Scale (CRS)}

The Craving Reactivity Scale derived from the modification of the Emotion Reactivity Scale (Nock, Wedig, Holmberg, \& Hooley, 2008). The modification was made by the researchers of this study, so that the instrument would exclusively focus on the emotion of craving. The CRS measures the intensity of craving reactivity in everyday life via a series of questions, such as "When I feel craving, it is the only thing I can think for a long time" and "My cravings go from zero to extreme in an instant". The possible responses to each of the 15 items of the questionnaire are placed in a Likert-type scale as follows: $0=$ Not at all like me, 1=A little like me, 2=Somewhat like me, 3=A lot like me, 4=Completely like me. The original scale had been normed on adolescents and young adults and shows strong internal consistency $(\alpha=.94)$, and convergent, divergent and criterion-related validity. In the context of this study, the Craving Reactivity Scale was used in order to investigate the effect of the proposed treatments in everyday life craving reactivity since low capacity to cope with craving is highly associated with relapse (Heinz, Beck, Grüsser, Grace, \& Wrase, 2009). 


\section{iii. Permissive Thoughts Questionnaire (ICT)}

The Permissive Thoughts Questionnaire (Hautekèete, Cousin, \& Graziani, 1999) was developed in order to measure the intensity of permissive thoughts on alcohol use. The Permissive Thoughts Questionnaire was normed on individuals suffering from alcohol dependence after research studies had revealed the existence of permissive thoughts in individuals with SUD which can be positively modified during CBT interventions (Tison \& Hautekèete, 1998). According to Hautekèete, Cousin and Graziani (1999) the ICT has shown to be a valid instrument for measuring changes in permissive thoughts during cognitive behavioural interventions. In the context of the present study, the ICT questions were duplicated to examine permissive thoughts on drug use, in addition to those concerning alcohol consumption that may indicate substance substitution tendencies. ICT was used in order to examine the impact of the proposed treatments on permissive thoughts on drug use and alcohol consumption that promote the maintenance of substance use and indicate high possibility for relapse (Romo \& Graziani, 2015).

\section{iv. Measurements of craving intensity during exposure to drug-conditioned cues}

The main objective of the study was to investigate the capacity of the proposed treatment modalities to reduce the intensity of craving during exposure to external drugconditioned cues. For this reason, participants watched in two groups a fifteen-minute film which involved drug-conditioned cues related to their substance of addiction. The films were chosen by the researchers based on the individual interviews of the pre-treatment period. They mainly included cues, such as drug paraphernalia, pharmacy signs and drug use and dealing, which were most commonly reported by participants as high-risk and strongly conditioned with drug use. All films involved these cues in similar frequency and context.

In order to maximize the validity and accuracy of participants' responses (Moeller, Konova, \& Goldstein, 2015), the researchers made specific clarifications to the participants 
before the craving measurements. First, craving was clearly defined as the intense desire to consume the substance of addiction, so that it would have the same meaning among participants and the researchers. Second, participants were encouraged to honestly evaluate their craving intensity. Third, participants were asked not to put any effort in being defensive or insensitive to the stimuli presented in the films, since the aim was to produce accurate craving assessments and track their therapeutic progress through the personalized results they would receive after the end of the study.

The film projections took place with muted sound in order to avoid influencing participants' affective functions as well as to eliminate the possibility of attention distraction induced by the music that accompanied the films. Each film was paused every five minutes and participants rated the intensity of their craving in a scale from 0 to 100 . Craving rates were also provided by participants immediately before and after the film projection. In case of persisting craving urges after the film projections, the researchers stayed with the participants until the urges completely faded out so as to eliminate any possibility for relapse.

In order to avoid potential learning effects, participants watched a different film during the baseline and post-treatment assessments. Half of the participants watched at baseline the film that the other half watched after the end of treatment and vice versa. In the follow-up evaluation, each group watched the film of the baseline measurements.

\section{v. Participation evaluation questionnaire (PEQ)}

The Participation Evaluation Questionnaire (PEQ) was developed by the researchers and aimed at receiving participants' feedback on the perceived benefits and/or negative effects of their participation in the study. It consists of 4 items, such as "Would you describe your participation in this study as positive or negative? Please describe the way in which it has influenced you", and "Has your participation in this study contributed to your treatment? 
If yes, please describe how". All four items included both a closed and an open-ended question. The PEQ was completed by participants during the post-treatment and follow-up measurements.

\section{Results}

The 21 male and 3 female individuals that participated in the study were all addicted to drugs. Their age ranged from 26 to 50 years old $(M=32.58, S D=5.79)$ and their time in treatment ranged from 3 to 18.5 months $(M=11.13, S D=4.07)$ in the ARGO program for addicted individuals. Ninety-six percent of them were addicted to heroin, while $4 \%$ were addicted to cannabis. In regards to prior musical experiences or training, $8.3 \%$ of the participants had received formal music training, $4.2 \%$ had been members of a music band, while another $4.2 \%$ had worked as disc-jockeys in the past.

Normality and outlier tests were conducted at baseline. No significant results outside the accepted ranges were reported in any of the dependent variables of the study.

Pearson product-moment bivariate correlations were computed at baseline to assess potential relationships between the dependent variables. The results are summarized in Table 1. Craving experienced during exposure to external drug-conditioned stimuli was positively correlated with everyday life craving reactivity $(r=.431, p=.035)$. As expected, it was also positively correlated with levels of depression symptoms $(r=.491, p=.015)$. The results indicate that the higher is the level of craving participants experienced during exposure to external drug-conditioned cues the more intense are depression symptoms and everyday craving reactivity. Permissive thoughts on drug use were positively correlated with everyday life craving reactivity $(r=.419, p=.042)$ and symptoms of depression $(r=.495, p=.014)$. These findings imply that the higher are the levels of permissive thoughts the more intense are depression symptoms and everyday life craving reactivity. Finally, everyday life craving 
reactivity was found to be positively correlated with depression $(r=.609, p=.002)$. This indicates that the higher is craving reactivity in everyday life the more intense are the symptoms of depression.

\subsection{Treatment outcomes}

We hypothesized that systematic desensitization combined with listening to two different kinds of relaxing music can reduce craving in response to external drug-conditioned cues significantly more than standard treatment alone. We also expected to observe a similar effect in permissive thoughts, craving reactivity in everyday life and depression symptoms. A mixed-design 3 x 3 ANOVA with Tukey's post hoc tests was conducted in order to investigate the within- and between-subjects effects in the dependent variables, as well as the interaction between time and group, across the three time points of the study $(\mathrm{T} 1=$ pretreatment, $\mathrm{T} 2=$ post-treatment and $\mathrm{T} 3=$ follow-up).

The results in the variable of craving experienced during exposure to cues conditioned with drug use showed that there was a significant difference across the three time points, $F$ $(2,42)=25.13, p<.001$, and significant differences between groups, $F(2,21)=5.57, p=$ .011. There was also a significant interaction between time and group, $F(4,42)=13.62, p<$ .001. Following up this interaction indicated that there was no significant difference between groups at baseline and that CTR only presented a significant increase from $\mathrm{T} 1$ to $\mathrm{T} 2$. In contrast, the mean scores of IMT and NIMT significantly decreased over time (see Figure 1a). These findings indicate that SD combined with IMT and NIMT can significantly reduce craving in response to external drug-conditioned cues.

In regards to permissive ideas for using drugs, significant differences across the three time points were observed, $F(2,42)=5.13, p=.01$. Although no statistically significant differences were found between groups, $F(2,21)=.36, p=.703$, a significant interaction 
between time and group was found, $F(4,42)=4.44, p=.004$. Post-hoc comparisons revealed no significant differences between groups at baseline. A significant increase was observed in the CTR condition from T1 to T2, as opposed to IMT that exhibited significant decreases over time. The NIMT condition presented decreases during the three time points of the study that did not reach statistical significance (see Figure 1b). These findings imply that SD combined with IMT appears to be more effective than SD combined with IMT in reducing permissive ideas for drug use.

The results on permissive ideas for alcohol use indicated that there was a significant difference across the three time points, $F(2,42)=4.5, p=.017$, but no significant differences between groups, $F(2,21)=.82, p=.921$. However, a significant interaction between time and group was found, $F(4,42)=3.29, p=.02$. Following up this interaction showed that there was no significant difference between groups at baseline and the control group did not significantly change over time. In contrast, the mean scores of IMT and NIMT significantly decreased over the three time points of the study, with IMT appearing to induce a slightly more pronounced effect (see Figure 1c). The results suggest that SD combined with both IMT and NIMT can elicit a positive effect on permissive ideas for alcohol use, with IMT appearing to be slightly more effective.

Analysis of craving reactivity results showed that there was a significant difference across the three time points, $F(2,42)=16.08, p<.001$, but no significant differences between groups, $F(2,21)=.102, p=.904$, nor a significant interaction between time and group, $F(4,42)=1.78, p=.151$. Post-hoc comparisons did not reveal any significant differences between groups at baseline or any significant change in the CTR condition during the study. In contrast, statistically significant decreases were observed within the other two conditions, with IMT exhibiting a slightly more intense effect than NIMT (see Figure 1d). 
These findings indicate that SD combined with IMT and NIMT may be capable of reducing craving reactivity in everyday life, with IMT appearing to elicit a slightly more pronounced effect.

In terms of depression symptoms, the results indicated that there were statistically significant changes across time, $F(2,42)=14.75, p<.001$, but no significant differences between groups were observed, $F(2,21)=.59, p=.564$. However, an interaction that approached statistical significance was observed between time and group, $F(4,42)=2.22, p$ $=.079$. Post-hoc comparisons showed that there was no significant difference between groups at baseline and the control group did not significantly change over time. In contrast, the mean scores of IMT and NIMT significantly decreased over the three time points of the study, with IMT presenting a slightly more pronounced effect (see Figure 1e). The results indicate that SD combined with IMT and NIMT induce a similar positive effect on depression symptoms, with IMT presenting a slightly stronger effect.

Finally, participants completed a research evaluation questionnaire in order to assess the impact that their participation in the study had upon them. Ninety-four percent of IMT and NIMT participants rated their participation as positive and therapeutically beneficial for them, while $6 \%$ reported that their experience was neither positive nor negative and did not have an important therapeutic effect on them. Among the participants who reported therapeutic benefits from participation in the study, 37.5\% underscored important craving reductions during exposure to high-risk situations and increased capacity to control craving urges in everyday life, $18.7 \%$ highlighted the benefit of reduced fear in response to drugconditioned cues, another $18.7 \%$ reported enhanced coping with negative emotions, while the remaining $25 \%$ stressed the improved coping skills for high-risk situations in everyday life. It is noteworthy that although the therapeutic process was often challenging for IMT and NIMT 
participants, there was a high rate of participation in the therapeutic sessions $(M=5.19, S D=$ 1.42) and no participants dropped out of the study. Furthermore, CTR participants emphasized the importance of the opportunity they were offered in the context of the study to follow their own progress concerning specific aspects of their drug addiction problem, such as their capacity to cope with craving, depression symptoms and permissive thoughts.

As time in treatment varied among participants, we conducted a one-way ANOVA to compare the baseline means in each dependent variable between participants who were in treatment for longer than eleven months and participants who were in treatment for less than eleven months, which was the average duration of treatment in the study sample. A one-way ANOVA was also conducted in order to compare the means of mean changes in the dependent variables between the participants who were in treatment for longer than eleven months and participants who were in treatment for less than eleven months, within each condition. The results showed that baseline craving intensity in response to drug-conditioned cues was significantly higher for the participants who were in treatment for less than eleven months $(p=.004)$. However, no statistically significant differences were found between these groups in the changes they experienced in this variable during the study.

\section{Discussion}

The present study shows that SD combined with IMT and NIMT can reduce craving in response to external cues conditioned with drug use. Given the fact that the effect was maintained one month after the end of treatment, the results of this study seem encouraging concerning the capacity of SD combined with IMT and NIMT to induce a positive effect on craving intensity during exposure to drug-conditioned cues. To the best of our knowledge, this is the first study to examine the therapeutic potential of music-assisted systematic desensitization in counterconditioning drug-related cues and reducing the implicated craving 
responses. This finding may prove to be useful for future cue-exposure studies that will shift their focus from cue extinction to cue counterconditioning, as already proposed by experts (Conklin \& Tiffany, 2002; Van Gucht et al., 2010). Future research with larger sample sizes is needed to further verify the findings of this study, shed light on the exact contribution of music to the specific therapeutic process and allow for generalization.

The research findings of the study indicated that only IMT was able to reduce permissive thoughts on drug use. This effect may be mediated by craving reactivity in everyday life which was also improved more for the IMT, since a positive correlation was observed at baseline between the two variables (see Figure 2). Consistent with this view, craving and permissive thoughts have been previously found to be highly associated (Ball, 2007; Dalley, Everitt, \& Robbins, 2011). Furthermore, baseline permissive thoughts on drug use were positively correlated with depression which also ameliorated slightly more for the IMT condition. These findings could explain why positive changes in permissive thoughts of NIMT participants did not reach statistical significance. Additionally, analysis of the results in permissive thoughts for alcohol use revealed similar changes with those observed in permissive thoughts for drug use, within IMT and NIMT. This finding implies that the IMT therapeutic modality may have a slightly stronger effect on potential substance substitution tendencies. This effect could be mediated by positive changes in permissive thoughts on drug use that are related to the amelioration of craving reactivity of everyday life. Consistent with this, previous studies have shown that craving for drugs is associated with craving for alcohol (Lubrano, Pacini, Giuntoli, \& Maremmani, 2002) and both are mediated by permissive thoughts (Romo and Graziani, 2015).

The assumption that reduced craving in response to stimuli conditioned with drug use and the associated everyday life craving reactivity amelioration may mediate positive 
changes in psychopathological components of drug addiction seems to also be verified by the results of the study on depression symptoms (see Figure 2). Baseline correlations revealed that depression symptoms of individuals with SUD are positively correlated with craving intensity experienced during exposure to drug-conditioned cues and with craving reactivity in everyday life. Amelioration of depression in both IMT and NIMT conditions appeared to follow the reduction of craving in response to cues conditioned with drug use. This effect appears to have been further enhanced by positive changes in everyday craving reactivity which could explain why IMT induced a more pronounced effect on depression symptoms than NIMT. These findings are consistent with prior studies which have linked craving with depression (Pierucci-Lagha et al., 2004; Wedekind et al., 2010) and have shown that high cue reactivity to drug-related stimuli induces increases in craving and depression which subside for a period of at least 6 weeks after cue-exposure therapy (Franken, de Haan, van der Meer, Haffmans, \& Hendriks, 1999). However, due to the confounded design of the study we cannot safely discriminate between the impact of music and that of systematic desensitization. Further research with a different study design is necessary for determining the impact of music on depression symptoms during cue-exposure therapy.

The results of the study also showed that baseline craving intensity in response to drug-conditioned cues was the only dependent variable of the study that was significantly higher for participants who were in treatment for less than eleven months than for those who were in treatment for longer than eleven months. Since no statistically significant differences were found between these two groups in the changes they experienced in this variable during the study, it seems safe to assume that the levels of craving of participants who were in treatment for longer than eleven months were still high enough to go through statistically significant changes. Prior research studies seem to verify this assumption as they have shown 
that cue reactivity is still high after long periods of treatment and that drug-related stimuli still induce considerable increases in craving that can subside after cue-exposure therapy (Crombag, Bossert, Koya, \& Shaham, 2008; Yokoyama et al., 2015)

Lastly, it must be mentioned that the possibility of prior music experiences or music training affecting the results of this study was taken into account. Analysis, however, did not reveal any statistically significant differences between participants who had and participants who had not any prior music experience or training.

Taken together, the findings of this study indicate that IMT and NIMT combined with SD are similarly effective in counterconditioning drug-related cues and reducing the involved craving responses. Importantly, participants' perceived therapeutic benefits from participation to the study are consistent with this finding as they mainly implicate significant craving reduction during exposure to high-risk situations, increased control over craving urges in everyday life, decreases of fear-induced confusion during exposure to drug-conditioned cues, enhanced coping with negative emotions, and improved coping skills for everyday high-risk situations. Of interest, overall results imply that IMT can induce a more pronounced effect than NIMT in the secondary variables of the study. Since this effect seems to be directly or indirectly related to the counterconditioning of cues conditioned with drug use, it would be interesting to see if longer periods of NIMT therapy could elicit an effect similar to that of IMT. Prior research findings seem to support this notion as they suggest that longer periods of cue-exposure therapy can induce more distinct therapeutic effects (Crombag, Bossert, Koya, \& Shaham, 2008).

As a conclusion, the present study investigated for the first time the beneficial effects of IMT and NIMT combined with SD in the reduction of craving in response to external cues conditioned with drug use. However, it must be said that larger scale trials are needed in 
order to verify the reported effects of IMT and NIMT combined with SD, as well as to allow the investigation of the roles of age and gender which were not examined in this study, since female individuals with SUD were highly outnumbered by males in our sample. Further research is also necessary in order to distinguish the effects of the music interventions from those of SD. Determining the potential contribution of music-assisted systematic desensitization and the functional basis of this therapeutic intervention in the treatment of drug addiction seems warranted.

\section{Conclusions}

It has been well documented that individuals with SUD often relapse after treatment when confronted with drug-related stimuli in real life (Emmelkamp \& Vedel, 2012). Therefore, part of therapeutic interventions for relapse prevention should target at providing individuals with SUD with alternative coping strategies so that they regain control over craving in their daily confrontations with drug-related cues. The results of our study indicate that two different modalities of listening to relaxing music combined with systematic desensitization may be useful complementary therapeutic interventions by reducing craving responses to drug-conditioned cues and facilitating the enhancement of coping skills for confronting high-risk situations. These findings imply that in the future, music-assisted systematic desensitization may prove to be a useful therapeutic technique for facilitating the containment of compulsive drug-seeking behaviours and for reacquiring cognitive control during exposure to high-risk situations that involve drug-conditioned cues. The considerably wide variety of music genres and the high preference of individuals with SUD for music (Aldridge \& Fachner, 2010) may play an important role in the development of the specific treatment modality. In conclusion, future research studies are encouraged in order to further 
investigate the potential contribution of music-assisted cue-exposure therapy in drug addiction treatment.

\section{Funding}

This study was supported by a grant from Grundtvig Education and Culture Lifelong Learning Program that was awarded to the first author in the form of a Grundtvig Assistantship (agreement contract number 2013-1-FR1-GRU11-50690). The funding source was fully informed of, but was not involved in the study design, collection, analysis and interpretation of data.

\section{Conflict of interest}

The authors report no conflict of interest.

\section{Acknowledgements}

The authors wish to thank the staff and direction of the ARGO Alternative Therapeutic Program for Addicted Individuals in the Psychiatric Hospital of Thessaloniki, in Greece. Special thanks are addressed to the director of the program Dr. Panagiotis Georgakas, as well as to Mr. Ioannis Ntolas, Mr. Apostolos Mpalasis, Mrs. Paraskevi Giannakaki, Mrs. Athina Vlahvei, Mr. Mihalis Milonas and Mrs. Angeliki Gkini for facilitating conducting this study. The authors would further wish to thank all participants for their willingness to participate in the study and their strong commitment to therapy. Finally, the authors would like to thank Grundtvig Education and Culture Lifelong Learning Program for financially supporting this study.

\section{References}

Aldridge, D., \& Fachner, J. (2010). Music therapy and addictions. London: Jessica Kingsley Publishers.

Aldridge, D., \& Fachner, J. (2006). Music and altered states - consciousness, transcendence, therapy and addictions. London: Jessica Kingsley Publishers.

Andrade, J., May, J., \& Kavanagh, D. K. (2008). Conscious and unconscious processes in human desire. Psyche, 15(2). 83-91. Retrieved from http://journalpsyche.org 
Baker, F. A., Gleadhill, L. M., \& Dingle, G. A. (2007). Music therapy and emotional exploration: Exposing substance abuse clients to the experiences of non-drug-induced emotions. The Arts in Psychotherapy, 34(4), 321-330. doi:10.1016/j.aip.2007.04.005

Ball, S. A. (2007). Cognitive-behavioural and schema-based models for the treatment of substance use disorders. In L. P. Riso, P. L. du Toit, D. J. Siein \& J. E. Young (Eds.), Cognitive schemas and core beliefs in psychological problems: A scientist practitioner guide (pp. 111-138). Washington, DC: American Psychological Association.

Bernatzky, G., Presch, M., Anderson, M., \& Panksepp, J. (2011). Emotional foundations of music as a non-pharmacological pain management tool in modern medicine. Neuroscience and Biobehavioral Reviews, 35(9), 1989-1999. doi: 10.1016/j.neubiorev.2011.06.005

Blood, A. J., \& Zatorre, R. J. (2001). Intensely pleasurable responses to music correlate with activity in brain regions implicated in reward and emotion. Proceedings of the National Academy of Sciences, USA, 98(20), 11818-11823. doi: 10.1073/pnas.191355898

Cevasco, A. M., Kennedy, R., \& Generally, N. R. (2005). Comparison of movement-to-music, rhythm activities, and competitive games on depression, stress, anxiety, and anger of females in substance abuse rehabilitation. Journal of Music Therapy, 42(1), 64-80. doi: $10.1093 / \mathrm{jmt} / 42.1 .64$

Conklin, C. A., \& Tiffany, S. T. (2002). Applying extinction research and theory to cue-exposure addiction treatments. Addiction, 97, 155-167. doi: 10.1046/j.1360-0443.2002.00014.x

Crombag, H. S., Bossert, J. M., Koya, E., \& Shaham, Y. (2008). Context-induced relapse to drug seeking: A review. Philosophical transactions of the Royal Society of London, Series B, 363(1507), 3233-3243. doi: 10.1098/rstb.2008.0090

Dalley, J. W., Everitt, B. J., \& Robbins, T. W. (2011). Impulsivity, compulsivity, and top-down cognitive control. Neuron - Cell, 69(4), 680-694. doi:10.1016/j.neuron.2011.01.020

Deady, M. (2009). A review of screening, assessment and outcome measures for drug and alcohol settings. Retrieved from Health Well website: http://www.thehealthwell.info/node/252189

de Niet, G., Tiemens, B., Lendemeijer, B., \& Hutschemaekers, G. (2009). Music-assisted relaxation to improve sleep quality: Meta-analysis. Journal of Advanced Nursing, 65(7), 1356-1364. doi: 10.1111/j.1365-2648.2009.04982.x

Derogatis, L. R. (1993). SCL-90-R: Administration, scoring \& procedures manual-ii for the r(evised) version and other instruments of the psychopathology rating scale series. Minneapolis: National Computer Systems.

Dingle, G. A., Kelly, P. J., Flynn, L. M., \& Baker, F. A. (2015). The influence of music on emotions and cravings in clients in addiction treatment: A study of two clinical samples. The Arts in Psychotherapy, 45, 18-25. doi:10.1016/j.aip.2015.05.005

Dingle, G. A., Gleadhill, L., \& Baker, F. A. (2008). Can music therapy engage patients in group cognitive behaviour therapy for substance abuse treatment? Drug and Alcohol Review, 27(2), 190-196. doi: 10.1080/09595230701829371

Emmelkamp, P. M., \& Vedel, E. (2012). Evidence-based treatments for alcohol and drug abuse: A practitioner's guide to theory, methods, and practice. London: Routledge.

Ersche, K. D., Turton, A. J., Pradhan, S., Bullmore, E. T., \& Robbins, T. W. (2010). Drug addiction endophenotypes: Impulsive versus sensation-seeking personality traits. Biological Psychiatry, 68(8), 770-773. doi: 10.1016/j.biopsych.2010.06.015

Franken, I. H., de Haan, H. A., van der Meer, C. W., Haffmans, P. M., \& Hendriks, V. M. (1999). Cue reactivity and effects of cue exposure in abstinent posttreatment drug users. Journal of Substance Abuse, 16(1), 81-85. doi:10.1016/S0740-5472(98)00004-X

Hall, W., Carter, A., \& Forlini, C. (2015). The brain disease model of addiction: Is it supported by the evidence and has it delivered on its promises?. The Lancet Psychiatry, 2(1), 105-110. doi: 10.1016/S2215-0366(14)00126-6

Hautekèete, M., Cousin, I., \& Graziani, P. (1999). Pensées dysfonctionnelles de l'alcoolodépendance: Un test du modèle de Beck: Schémas anticipatoire, soulageant et permissif. [Dysfunctional thoughts of alcohol dependence: A Beck model test: Anticipatory, relief-orientated and 
permissive thoughts]. Journal de Thérapie Comportementale et Cognitive, 9(4), 108-112. doi: JTCC-12-1999-9-4-1155-1704-101019-ART50

Havermans, R. C., \& Jansen, A. T. (2003). Increasing the efficacy of cue exposure treatment in preventing relapse of addictive behavior. Addictive Behaviors, 28(5), 989-994. doi:10.1016/S0306-4603(01)00289-1

Heinz, A., Beck, A., Grüsser, S. M., Grace, A. A., \& Wrase, J. (2009). Identifying the neural circuitry of alcohol craving and relapse vulnerability. Addiction Biology, 14(1), 108-118. doi: 10.1111/j.1369-1600.2008.00136.x

Hone-Blanchet, A., Wensing, T., \& Fecteau, S. (2014). The use of virtual reality in craving assessment and cue-exposure therapy in substance use disorders. Frontiers in Human Neuroscience, 8, 844. doi: 10.3389/fnhum.2014.00844

Horowitz, L.M., Rosenberg, S.E., Baer, B.A., Ureno, G. \& Villasenor, V.S. (1988). Inventory of interpersonal problems: Psychometric properties and clinical applications. Journal of Consulting and Clinical Psychology, 56(6), 885-892. Retrieved from http://www.apa.org/pubs/journals/ccp

Kemper, K. J., \& Danhauer, S. C. (2005). Music as therapy. Southern Medical Journal, 98(3), 282288. Retrieved from http://sma.org/smj-home

Labbe, E., Schmidt, N., Babin, J., \& Pharr, M. (2007). Coping with stress: The effectiveness of different types of music. Applied Psychophysiology and Biofeedback, 32(3-4), 163-168. doi: 10.1007/s10484-007-9043-9

Landry, J. M. (2014). Physiological and psychological effects of a Himalayan singing bowl in meditation practice: A quantitative analysis. American Journal of Health Promotion, 28(5), 306-309. doi: 10.4278/ajhp.121031-ARB-528

Lee, E., Ku, J., Jung, Y. C., Lee, H., An, S. K., Kim, K. R., . . . Namkoong, K. (2013). Neural evidence for emotional involvement in pathological alcohol craving. Alcohol and Alcoholism, 48(3), 288-294. doi: 10.1093/alcalc/ags130.

Levy, N. (2013). Addiction is not a brain disease (and it matters). Frontiers in psychiatry, 4, 24. doi: 10.3389/fpsyt.2013.00024

Lubrano, S., Pacini, M., Giuntoli, G., \& Maremmani, I. (2002). Is craving for heroin and alcohol related to low methadone dosages in methadone maintained patients? Heroin Addiction and Related Clinical Problems, 4(2), 11-17. Retrieved from http://www.heroinaddictionrelatedclinicalproblems.org/harcp-archives-doi-articles.php

Manley, D. S. (2008). Acceptability and applicability of cue exposure therapy as a relapse prevention intervention for individuals who have substance misuse and mental health problems. Mental Health and Substance Use, 1(2), 172-184. doi:10.1080/17523280802020099

May, J., Kavanagh, D. J., \& Andrade, J. (2015). The elaborated intrusion theory of desire: A 10-year retrospective and implications for addiction treatments. Addictive behaviors, 44, 2934. doi: 10.1016/j.addbeh.2014.09.016

Mays, K. L., Clark, D. L., \& Gordon, A. J. (2008). Treating addiction with tunes: A systematic review of music therapy for the treatment of patients with addictions. Substance Abuse, 29(4), 51-59. doi: $10.1080 / 08897070802418485$

McHugh, R. K., Park, S., \& Weiss, R. D. (2014). Cue-induced craving in dependence upon prescription opioids and heroin. American Journal on Addictions, 23(5), 453-458. doi: 10.1111/j.1521-0391.2014.12129.x

Menon, V., \& Levitin, D. J. (2005). The rewards of music listening: Response and physiological connectivity of the mesolimbic system. Neuroimage, 28(1), 175-184. doi:10.1016/j.neuroimage.2005.05.053

Moeller, S. J., Konova, A. B., \& Goldstein, R. Z. (2015). Multiple ambiguities in the measurement of drug craving. Addiction, 110(2), 205-206. doi: 10.1111/add.12726

Myers, K. M., \& Carlezon, W. A. Jr. (2010). Extinction of drug- and withdrawal-paired cues in animal models: Relevance to the treatment of addiction. Neuroscience \& Biobehavioral Reviews, 35(2), 285-302. doi: 10.1016/j.neubiorev.2010.01.011 
Nock, M. K., Wedig, M. M., Holmberg, E. B., \& Hooley, J. M. (2008). The emotion reactivity scale: Development, evaluation, and relation to self-injurious thoughts and behaviors. Behavior Therapy, 39(2), 107-116. doi: 10.1016/j.beth.2007.05.005

Panksepp, J. (2007). Neurologizing the psychology of affects: How appraisal-based constructivism and basic emotion theory can coexist. Perspectives on Psychological Science, 2(3), 281-296. doi: 10.1111/j.1745-6916.2007.00045.x

Pavlov, I. P. (1927/1960). Conditional reflexes. New York: Dover Publications.

Phipps, M. A., Carroll, D. L., \& Tsiantoulas, A. (2010). Music as a therapeutic intervention on an inpatient neuroscience unit. Complementary Therapies in Clinical Practice, 16(3), 138-142. doi: 10.1016/j.ctcp.2009.12.001

Pierucci-Lagha, A., Feinn, R., Modesto-Lowe, V., Swift, R., Nellissery, M., Covault, J., \& Kranzler, H. R. (2004). Effects of rapid tryptophan depletion on mood and urge to drink in patients with co-morbid major depression and alcohol dependence. Psychopharmacology, 171(3), 340-348. doi: 10.1007/s00213-003-1588-6

Ramirez, J. J., Monti, P. M., \& Colwill, R. M. (2015). Brief and extended alcohol-cue-exposure effects on craving and attentional bias. Experimental and Clinical Psychopharmacology, 23(3), 159-167. doi: 10.1037/pha0000018

Romo, L., \& Graziani, P. (2015). Surmonter un problème avec l'alcool. [Getting over alcohol problems]. Paris, France: Dunod.

Saban, A., Flisher, A., Laubscher, R., London, L., \& Morojele, N. (2014). The association between psychopathology and substance use: Adolescent and young adult substance users in inpatient treatment in Cape Town, South Africa. The Pan African Medical Journal, 17(Suppl 1), 8. doi: 10.11694/pamj.supp.2014.17.1.3044

Sakamoto, M., Ando, H., \& Tsutou, A. (2013). Comparing the effects of different individualized music interventions for elderly individuals with severe dementia. International Psychogeriatrics, 25(5), 775-784. doi: 10.1017/S1041610212002256

Salamon, E., Kim, M., Beaulieu, J., \& Stefano, G. B. (2003). Sound therapy induced relaxation: Down regulating stress processes and pathologies. Medical Science Monitor, 9(5), 96-101. Retrieved from http://www.medscimonit.com

Salimpoor, V. N., Benovoy, M., Larcher, K., Dagher, A., \& Zatorre, R. J. (2011). Anatomically distinct dopamine release during anticipation and experience of peak emotion to music. Nature Neuroscience, 14(2), 257-262. doi:10.1038/nn.2726

Salimpoor, V. N., Benovoy, M., Longo, G., Cooperstock, J. R., \& Zatorre, R. J. (2009). The rewarding aspects of music listening are related to degree of emotional arousal. PLoS One, 4(10), 7487. doi: 10.1371/journal.pone.0007487

Serafin, S., Wilkerson, C., \& Smith, J. O. (2002, September). Modelling bowl resonators using circular wave guide networks. Paper presented in the 5th International Conference on Digital Audio Effects, Hamburg, Germany.

Sheldon, S., Kashdan, L., \& Steger, M. (2011). Designing the future of positive psychology: Taking stock and moving forward. New York: Oxford University Press.

Short, A. D., \& Dingle, G. A. (2015). Music as an auditory cue for emotions and cravings in adults with substance use disorders. Psychology of Music, Advance online publication. Retrieved from http://pom.sagepub.com

Smith, J. C., \& Joyce, C. A. (2004). Mozart versus new age music: Relaxation states, stress, and ABC relaxation theory. Journal of Music Therapy, 41(3), 215-224. doi: 10.1093/jmt/41.3.215

Stathopoulou, G. (2009). Gender, emotional intolerance, and mood-related cravings for substance use in treatment-seeking opiate-dependent patients (Unpublished doctoral dissertation). Boston University, Boston.

Tiffany, S. T. (2010). Drug craving and affect. In J. D. Kassel (Ed), Substance abuse and emotion (pp. 83-108). Washington, DC: American Psychological Association.

Tison, P., \& Hautekeete, M. (1998). Mise en évidence de schémas cognitifs dysfonctionnels chez des toxicomanes. [Revealing dysfunctional cognitive schemas in drug-addicted individuals]. Journal de thérapie comportementale et cognitive, 8(2), 43-49. doi: JTCC-07-1998-8-2-11551704-101019-ART68 
Van Gucht, D., Baeyens, F., Hermans, D., \& Beckers, T. (2013). The inertia of conditioned craving. Does context modulate the effect of counterconditioning?. Appetite, 65, 51-57. doi: 10.1016/j.appet.2013.01.019

Van Gucht, D., Baeyens, F., Vansteenwegen, D., Hermans, D., \& Beckers, T. (2010). Counterconditioning reduces cue-induced craving and actual cue-elicited consumption. Emotion, 10(5), 688-695. doi: 10.1037/a0019463

Wedekind, D., Herchenhein, T., Kirchhainer, J., Bandelow, B., Falkai, P., Engel, K., \& HavemannReinecke, U. (2010). Serotonergic function, substance craving, and psychopathology in detoxified alcohol-addicted males undergoing tryptophan depletion. Journal of Psychiatric Research, 44(16), 1163-1169. doi: 10.1016/j.jpsychires.2010.04.002

Wise, R. A., \& Koob, G. F. (2014). The development and maintenance of drug addiction. Neuropsychopharmacology, 39(2), 254-262. doi: 10.1038/npp.2013.261

World Health Organization. (1990). International Statistical Classification of Diseases and Related Health Problems (10th rev.). Geneva: World Health Organization.

Volkow, N. D., \& Morales, M. (2015). The brain on drugs: From reward to addiction. Cell, 162(4), 712-725. doi: 10.1016/j.cell.2015.07.046

Yokoyama, A., Matsushita, S., Toyama, T., Nakayama, H., Takimura, T., Kimura, M., ... \&

Yokoyama, T. (2015). Relapse prevention program consisting of coping skills training, cue exposure treatment, and letter therapy for Japanese alcoholic men who relapsed after standard cognitive-behavioral therapy. Nihon Arukoru Yakubutsu Igakkai zasshi, 50(2), 88-103. 
Table 1. Presentation of baseline correlations between the dependent variables of the study.

\begin{tabular}{|c|c|c|c|c|c|c|}
\hline Var & able & $\begin{array}{l}\text { Craving to } \\
\text { external }\end{array}$ & $\begin{array}{c}\text { ICT } \\
\text { drugs }\end{array}$ & CRS & $\begin{array}{c}\text { SCL 90R } \\
\text { depression }\end{array}$ & $\begin{array}{c}\text { ICT } \\
\text { alcohol }\end{array}$ \\
\hline & Pearson's & 1 & .246 & $.431 *$ & $.491 *$ & .361 \\
\hline Craving to & Sig. (2-tailed) & . & .246 & .035 & .015 & .083 \\
\hline & & 24 & 24 & 24 & 24 & 24 \\
\hline & Pearson's & & 1 & $419 *$ & $495 *$ & \\
\hline ICT (drugs) & Sig. (2-tailed) & & & (3) & 014 & 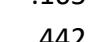 \\
\hline & $\mathrm{N}$ & & 24 & 24 & 24 & 24 \\
\hline & Pearson's & & & 1 & 60१** & 177 \\
\hline CRS & Sig. (2-tailed) & & & 1 & 002 & 408 \\
\hline & $\mathrm{N}$ & & & 24 & 24 & 24 \\
\hline & Pearson's & & & & 1 & 214 \\
\hline SCL $90 \mathrm{R}$ & Sig. (2-tailed) & . & & & $\perp$ & $\begin{array}{r}.214 \\
315\end{array}$ \\
\hline depression & $\mathrm{N}$ & & & & 24 & 24 \\
\hline & Pearson's & & & & & 1 \\
\hline ICT alcohol & Sig. (2-tailed) & & . & & & \\
\hline & $\mathrm{N}$ & & & & & 24 \\
\hline
\end{tabular}

*Correlation is significant at 0.05 (2-tailed)

**Correlation is significant at 0.01 (2-tailed) 


\section{FIGURES}

(a)

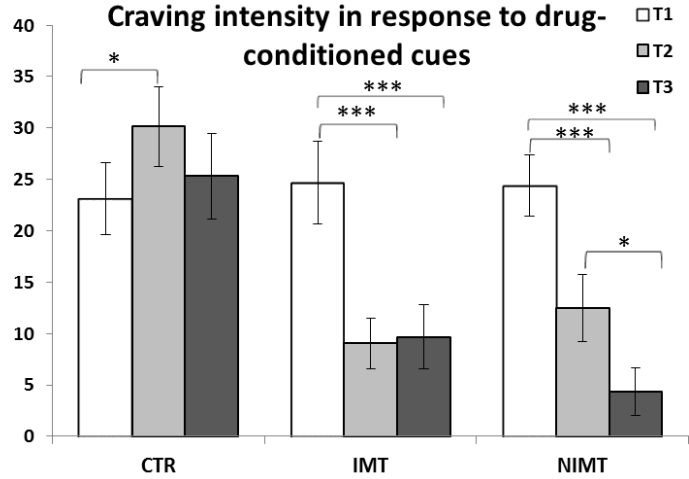

(c)

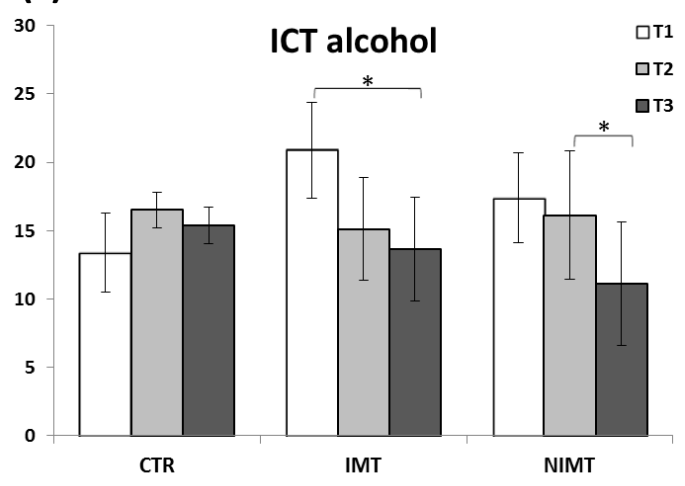

(e) (b)

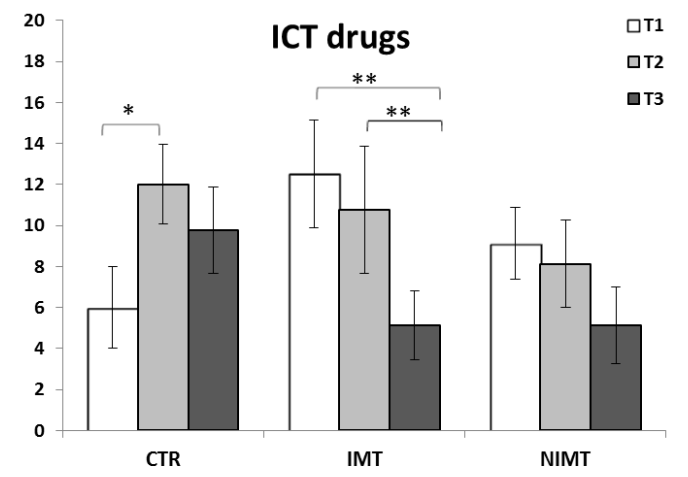

(d)

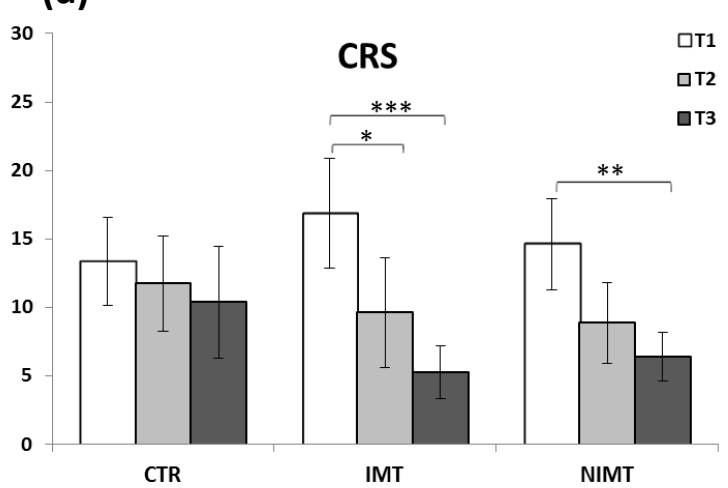

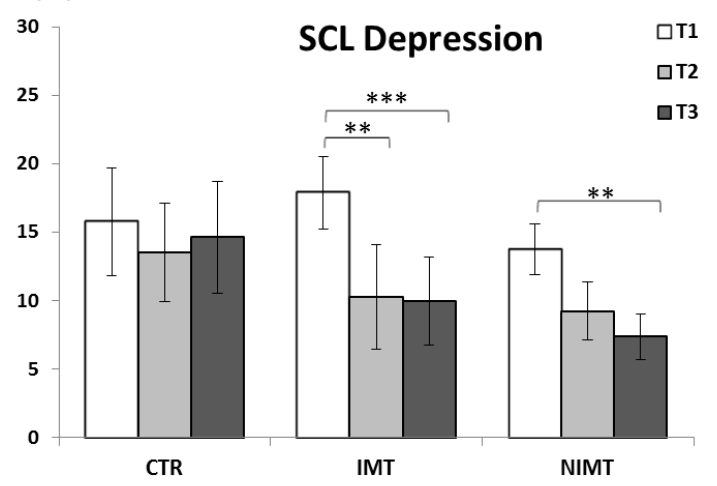

Figure 1. Graphic representation of IMT, NIMT and CTR changes that took place during the three time points of the study. Single asterisks $\left(^{*}\right)$ indicate significant changes at the .05 level, double asterisks $(* *)$ indicate significant changes at the .01 level, and triple asterisks $(* * *)$ indicate significant changes at the .001 level. 


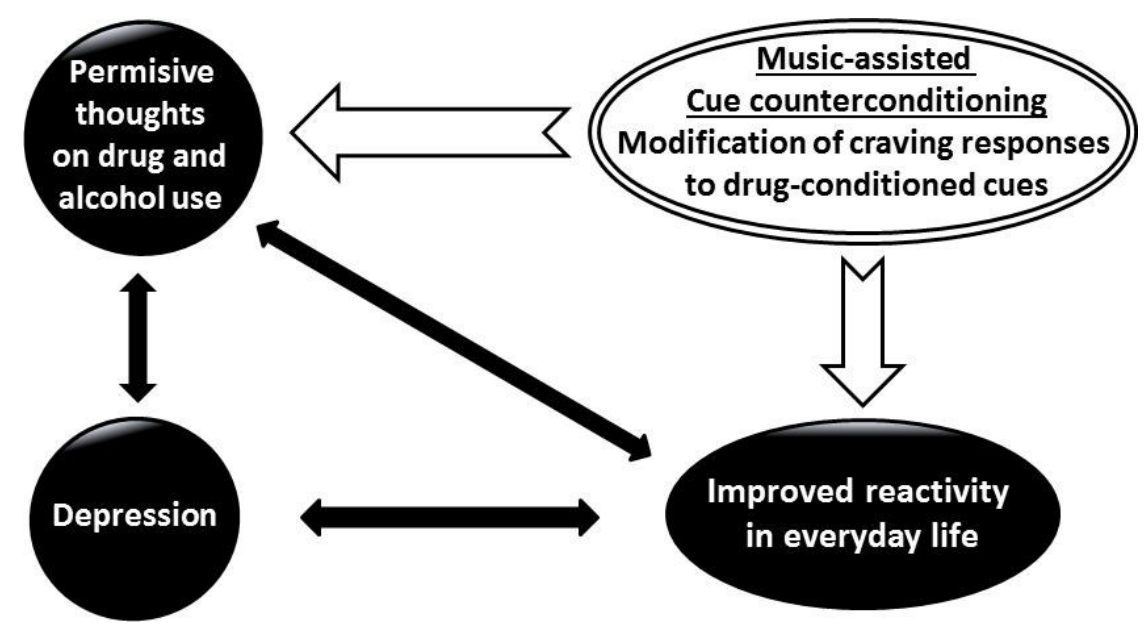

Figure 2. Illustration of the mechanisms by which IMT and NIMT treatment modalities appeared to influence the dependent variables of the study. The arrows indicate single or reciprocal influence between variables. 